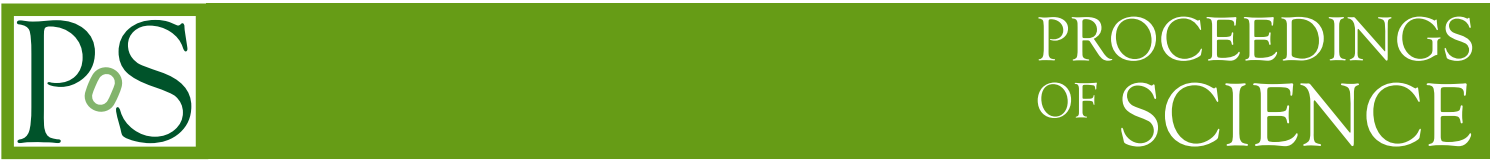

\title{
Belle II Vertex Detector Performance
}

T. Bilka ${ }^{* d d}$, F. Abudinén ${ }^{n}$, K. Ackermann ${ }^{n}$, P. Ahlburg ${ }^{e}$, H. Aihara ${ }^{a c}$, M. Albalawi ${ }^{n}$, O. Alonso ${ }^{a i}$, L. Andricek ${ }^{o}$, R. Ayad ${ }^{a h}$, T. Aziz ${ }^{u}$, V. Babu ${ }^{h}$, S. Bacher ${ }^{a g}$, S. Bahinipati ${ }^{p}$, Y. Bai ${ }^{m}$, E. Barberio ${ }^{a}$, Ti. Baroncelli ${ }^{a}$, To. Baroncelli ${ }^{a}$, A. K. Basith ${ }^{q}$, G. Batignani ${ }^{v, w}$, A. Bauer ${ }^{b}$, P. K. Behera ${ }^{q}$, V. Bertacchi ${ }^{v}, w$, S. Bettarini ${ }^{v, w}$, B. Bhuyan ${ }^{r}$, R. Blanco ${ }^{j}$, F. Bosi ${ }^{w}$, M. Boronat ${ }^{a} k$, L. Bosisio ${ }^{x, y}$, A. Bozek ${ }^{a g}$, F. Buchsteiner ${ }^{b}$, C. Camien $^{h}$, A. Caldwell $^{n}$, G. Caria ${ }^{a}$, G. Casarosa ${ }^{v, w}$, M. Ceccanti ${ }^{w}$, D. Červenkov ${ }^{d}$, V. Chekelian $^{n}$, T. Czank ${ }^{a b}$, N. Dash ${ }^{p}$, M. De Nuccio ${ }^{v, w}$, B. Deschamps ${ }^{e}$, A. Dieguez $^{a i}$, J. Dingfelder ${ }^{e}$,

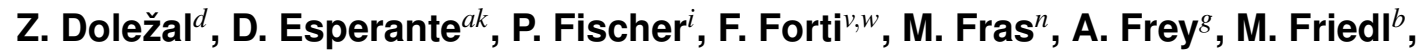
J. Fuster ${ }^{a k}$, M. Gabriel ${ }^{n}$, K. Gadow ${ }^{h}$, U. Gebauer ${ }^{g}$, L. Germic ${ }^{e}$, T. Gessler ${ }^{f}$, D. Getzkow ${ }^{f}$, L. Gioi ${ }^{n}$, A. Glazov ${ }^{h}$, B. Gobbo ${ }^{y}$, P. Gomis ${ }^{a k}$, J. A. M. Grimaldo ${ }^{a c}$, K. Hara ${ }^{a d}$, M. Heck ${ }^{j}$, T. Hemperek ${ }^{e}$, M. Hensel ${ }^{o}$, T. Higuchi ${ }^{z}$, M. Hoek ${ }^{k}$, C. Irmler ${ }^{b}$, A. Ishikawa ${ }^{a b}$, I. Jaegle ${ }^{a l}$, H. B. Jeon ${ }^{a e}$, C. Joo ${ }^{z}$, M. Kaleta ${ }^{a g}$, J. Kandra ${ }^{d}$, N. Kambara ${ }^{a d}$, K. H. Kang ${ }^{a e}$, P. Kapusta ${ }^{a g}$, C. Kiesling ${ }^{n}$, B. Kisielewski ${ }^{a g}$, D. Kittlinger $^{n}$, D. Klose ${ }^{o}$, P. Kodyš ${ }^{d}$, C. Koffmane ${ }^{o}$, T. Kohriki ${ }^{a d}$, S. Koike ${ }^{C, a d}$, I. Komarov ${ }^{y}$, I. Konorov ${ }^{m}$, S. Krivokuca ${ }^{o}$, H. Krüger ${ }^{e}$, T. Kuhr ${ }^{l}$, W. Kühn ${ }^{f}$, M. Kumar ${ }^{s}$, R. Kumar ${ }^{t}$, P. Kvasnička ${ }^{d}$, C. La Licata ${ }^{x, y}$, C. Lacasta ${ }^{a k}$, K. Lalwani $^{s}$, L. Lanceri ${ }^{x, y}$, J. S. Lange ${ }^{f}$, K. Lautenbach ${ }^{f}$, J. Y. Lee ${ }^{a f}$, S. C. Lee ${ }^{a e}$, U. Leis ${ }^{n}$, P. Leit ${ }^{n}$, D. Levit ${ }^{m}$, Y. Li ${ }^{a n}$, J. Libby $^{q}$, G. Liemann ${ }^{o}$, Z. Liu ${ }^{c}$, T. Lueck ${ }^{v, w}$, F. Lütticke ${ }^{e}$, L. Macharski ${ }^{h}$, P. Mammini ${ }^{w}$, C. Mariñas ${ }^{e}$, A. Martini ${ }^{v, w}$, S. N. Mayekar ${ }^{u}$, S. Mccarney ${ }^{n}$, G. B. Mohanty ${ }^{u}$, T. Morii' ${ }^{z}$, H. G. Moser ${ }^{n}$, D. Moya ${ }^{a j}$, F. J. Mueller ${ }^{h}$, F. Müller ${ }^{n}$, D. Münchow ${ }^{f}$, K. R. Nakamura ${ }^{a d}$, H. Nakayama ${ }^{a d}$,Z. Natkaniec ${ }^{a g}$, C. Niebuhr ${ }^{h}$, J. Ninkovic ${ }^{o}$, Y. Onuki ${ }^{a c}$, W. Ostrowicz ${ }^{a g}$, U. Packheiser ${ }^{h}$, A. Paladino ${ }^{z}$, E. Paoloni ${ }^{v, w}$, H. Park ${ }^{a e}$, B. Paschen ${ }^{e}$, S. Paul ${ }^{m}$, I. Peric ${ }^{j}$, F. Poblotzki ${ }^{h}$, K. Prasanth ${ }^{u}$, C. Praz ${ }^{h}$, A. Profeti ${ }^{w}$, A. Rabusov ${ }^{m}$, I. Rashevskaya ${ }^{A, y}$, K. K. Rao ${ }^{u}$, S. P. Reiter ${ }^{f}$, Resmi P. K. $^{q}$, R. Richter ${ }^{o}$, M. Ritter ${ }^{l}$, M. Ritzert ${ }^{i}$, G. Rizzo ${ }^{v, w}$, M. Rozanska $^{a g}$, S. Rummel ${ }^{l}$, D. Sahoo ${ }^{u}$, J. G. Sanchez ${ }^{a j}$, L. Santelj ${ }^{a m}$, J. Sasaki $^{a c}$, N. Sato ${ }^{a d}$, B. Scavino ${ }^{k}$, G. Schaller ${ }^{o}$, M. Schnecke ${ }^{o}$, F. Schopper ${ }^{o}$, H. Schreeck ${ }^{g}$, S. Schultschik ${ }^{b}$, C. Schwanda ${ }^{b}$, B. Schwenker ${ }^{g}$, R. Sedlmeyer ${ }^{n}$, C. Sfienti ${ }^{k}$, F. Simon $^{n}$, S. Skambraks ${ }^{n}$, Y. Soloviev ${ }^{h}$, B. Spruck ${ }^{k}$, R. Stever $^{h}$, U. Stolzenberg ${ }^{g}$, J. Stypula $^{a g}$, J. Suzuki ${ }^{a d}$, E. Tafelmayer ${ }^{o}$, M. Takahashi ${ }^{h}$, S. Tanaka ${ }^{a d}$, H. Tanigawa ${ }^{a c}$, G. N. Taylor ${ }^{a}$, R. Thalmeier ${ }^{b}$, T. Tsuboyama ${ }^{a d}$, P. Urquijo ${ }^{a}$, I. Vila ${ }^{a j}$, A. L. Virto ${ }^{a j}$, L. Vitale ${ }^{x, y}$,

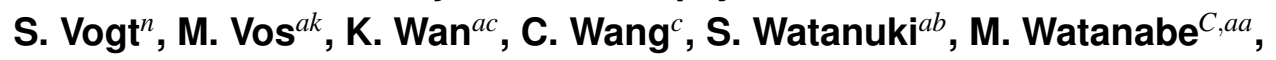
I. J. Watson ${ }^{a c}$, J. Webb $^{a}$, N. Wermes ${ }^{e}$, C. Wessel ${ }^{e}$, J. Wiechczynski ${ }^{a g}$, P. Wieduwilt ${ }^{g}$, S. Williams ${ }^{a}$, H. Windel ${ }^{n}$, H. Ye ${ }^{h}$, H. Yin ${ }^{b}$, L. Zani ${ }^{v, w}$, J. Zhao 
(Belle II DEPFET, PXD, and SVD Collaborations)

a School of Physics, University of Melbourne, Melbourne, Victoria 3010, Australia

${ }^{b}$ Institute of High Energy Physics, Austrian Academy of Sciences, 1050 Vienna, Austria

${ }^{c}$ Institute of High Energy Physics, CAS, 19B Yuquan Road, Shijingshan District, Beijing, China

${ }^{d}$ Faculty of Mathematics and Physics, Charles University, 12116 Prague, Czech Republic

e University of Bonn, 53115 Bonn, Germany

${ }^{f}$ Justus-Liebig-Universität Gießen, 35392 Gießen, Germany

${ }^{g}$ II. Physikalisches Institut, Georg-August-Universität Göttingen, 37073 Göttingen, Germany

${ }^{h}$ Deutsches Elektronen-Synchrotron, 22607 Hamburg, Germany

${ }^{i}$ Institute for Computer Engineering, Heidelberg University, 69117 Heidelberg, Germany

${ }^{j}$ Karlsruhe Institute of Technology, Hermann-von-Helmholtz-Platz 1, 76344

Eggenstein-Leopoldshafen, Karlsruhe, Germany

${ }^{k}$ Johannes Gutenberg University Mainz, 55099 Mainz, Germany

${ }^{l}$ Ludwig Maximilians University, 80539 Munich, Germany

${ }^{m}$ Technical University of Munich, Arcisstrasse 21, D-80333 Munich, Germany

${ }^{n}$ Max Planck Institute for Physics, D-80805 Munich, Germany

${ }^{o}$ Halbleiterlabor der Max-Planck-Gesellschaft, Otto-Hahn-Ring 6, D-81739 Munich, Germany

${ }^{p}$ Indian Institute of Technology Bhubaneswar, Satya Nagar, India

${ }^{q}$ Indian Institute of Technology Madras, Chennai 600036, India

${ }^{r}$ Indian Institute of Technology Guwahati, Assam 781039, India

${ }^{s}$ Malaviya National Institute of Technology Jaipur, Jaipur 302017, India

${ }^{t}$ Punjab Agricultural University, Ludhiana 141004, India

" Tata Institute of Fundamental Research, Mumbai 400005, India

${ }^{v}$ Dipartimento di Fisica, Università di Pisa, I-56127 Pisa, Italy

${ }^{w}$ INFN Sezione di Pisa, I-56127 Pisa, Italy

${ }^{x}$ Dipartimento di Fisica, Università di Trieste, I-34127 Trieste, Italy

${ }^{y}$ INFN Sezione di Trieste, I-34127 Trieste, Italy, ${ }^{A}$ presently at TIFPA - INFN, I-38123 Trento, Italy

${ }^{z}$ Kavli Institute for the Physics and Mathematics of the Universe (WPI), University of Tokyo, Kashiwa 277-8583, Japan

aa Department of Physics, Niigata University, Niigata 950-2181, Japan, ${ }^{B}$ presently at Nippon Dental University, Niigata 951-8580, Japan

${ }^{a b}$ Department of Physics, Tohoku University, Sendai 980-8578, Japan

ac Department of Physics, University of Tokyo, Tokyo 113-0033, Japan

${ }^{a d}$ High Energy Accelerator Research Organization (KEK), Tsukuba 305-0801, Japan, ${ }^{C}$ deceased

ae Department of Physics, Kyungpook National University, Daegu 702-701, Kored Department of Physics and Astronomy, Seoul National University, Seoul 151-742, Korea

ag H. Niewodniczanski Institute of Nuclear Physics, Krakow 31-342, Poland

${ }^{a h}$ Department of Physics, Faculty of Science, University of Tabuk, Tabuk 71451, Saudi Arabia

ai University of Barcelona, C/Marti Franques, 1., 08028-Barcelona, Spain

${ }^{a j}$ Instituto de Fisica de Cantabria (CSIC-UC), Avd. de los Castros s/n, 39005 Santander, Spain

${ }^{a k}$ IFIC (UVEG/CSIC), Edificio Institutos de Investigación Apartado de Correos 22085 E-46071 Valencia, Spain

al University of Florida, Department of Physics, P.O. Box 118440, Gainesville, FL 32611, USA

am J. Stefan Institute, Faculty of Mathematics and Physics, University of Ljubljana, 1000 Ljubljana, Slovenia

an Peking University, Department of Technical Physics, Beijing 100871, China 
The Belle II experiment at the SuperKEKB accelerator (KEK, Tsukuba, Japan) collected its first $e^{+} e^{-}$collision data in the spring 2019. The aim of accumulating a 50 times larger data sample than Belle at KEKB, a first generation $B$-Factory, presents substantial challenges to both the collider and the detector, requiring not only state-of-the-art hardware, but also modern software algorithms for tracking and alignment. The broad physics program requires excellent performance of the vertex detector, which is composed of two layers of DEPFET pixels and four layers of double sided-strip sensors. In this contribution, an overview of the vertex detector of Belle II and our methods to ensure its optimal performance, are described, and the first results and experiences from the first physics run are presented.

The 28th International Workshop on Vertex Detectors - Vertex2019

13-18 October, 2019

Lopud, Croatia

\footnotetext{
* Speaker.

${ }^{\dagger}$ for the Belle II DEPFET, PXD, and SVD Collaboration
} 


\section{Introduction}

The Belle II detector at the SuperKEKB accelerator (KEK, Tsukuba, Japan) [1] is an upgrade of the Belle experiment at the KEKB collider and is a part of a new generation of $B$-Factories. The design luminosity of SuperKEKB is 40 times larger than that achieved by KEKB, which is the current world record; this luminosity will allow Belle II to collect a 50 times larger data sample than Belle. Belle II has a broad physics program at the luminosity frontier, not only related to $B$ mesons, but also charm hadrons, $\tau$ leptons, spectroscopy, and dark-matter searches.

Belle II is a precision experiment, looking for small deviations from the Standard Model, which would indicate New Physics. To be able to observe such deviations, both statistical and systematic uncertainties have to be reduced. The increased statistics due to the accelerator upgrade, must be complemented by improved sub-detectors, algorithms and calibrations, to suppress the systematic errors, which might eventually dominate the total uncertainties.

Belle II had its first $e^{+} e^{-}$data taking with an almost complete ${ }^{1}$ vertex detector from March to July 2019 and collected about $6.5 \mathrm{fb}^{-1}$ of data at a center-of-mass energy equal to the mass of the $\Upsilon(4 S)$ resonance or close to it for continuum background studies. Despite the limited statistics, this operational period allows a better understanding of the detector, such that the reconstruction and alignment can be tuned for optimal performance.

We will give a short overview of the vertex detector in Sec. 2 and the tracking and vertexing software, including our alignment method in Sec. 3. The results obtained during a commissioning run in 2018 and cosmic ray tests in 2019, as well as the first results from $e^{+} e^{-}$collisions, are presented in Sec. 4.

\section{Belle II Vertex Detector}

Vertexing (or decay-vertex reconstruction) has a special role at the $B$-Factories. For example, measurements of time-dependent $C P$ violation require a precise determination of the distance between decay vertices of neutral $B$ mesons. The reduced boost of the center-of-mass system at SuperKEKB means a reduction of this distance on average, which is more than compensated by an approximately two times better impact parameter resolution at Belle II with respect to Belle. Also the decays of $B$ mesons have a soft momentum spectrum of final-state particles with $p<2.5 \mathrm{GeV} / c$ and average momentum well under $0.5 \mathrm{GeV} / c$, which means that the resolution becomes limited by multiple-scattering effects. Therefore the vertex detector must be very lightweight, with most of its support structures and services placed outside of the acceptance.

The vertex detector (VXD) is made of two layers of PiXel Detector (PXD) with pixel sensors and four layers of Silicon Vertex Detector (SVD), with double-sided strip sensors. One of the VXD half-shells, before completion and installation, is depicted in Fig. 1.

PXD [2] uses DEPFET modules thinned to $75 \mu \mathrm{m}\left(0.2 \% X_{0} /\right.$ layer $)$. The first layer with 12 sensors (pixel size $50 \mu \mathrm{m} \times 55-60 \mu \mathrm{m}$ ) is placed only $14 \mathrm{~mm}$ from the interaction point. In its design configuration, about eight million pixels are used for precise vertex reconstruction. For initial physics operation phase, only four sensors (pixel size $50 \mu \mathrm{m} \times 70-85 \mu \mathrm{m}$ ) are installed in the second layer.

\footnotetext{
${ }^{1}$ The second layer of the pixel detector is only partially equipped, because the sensors were not ready on time.
} 


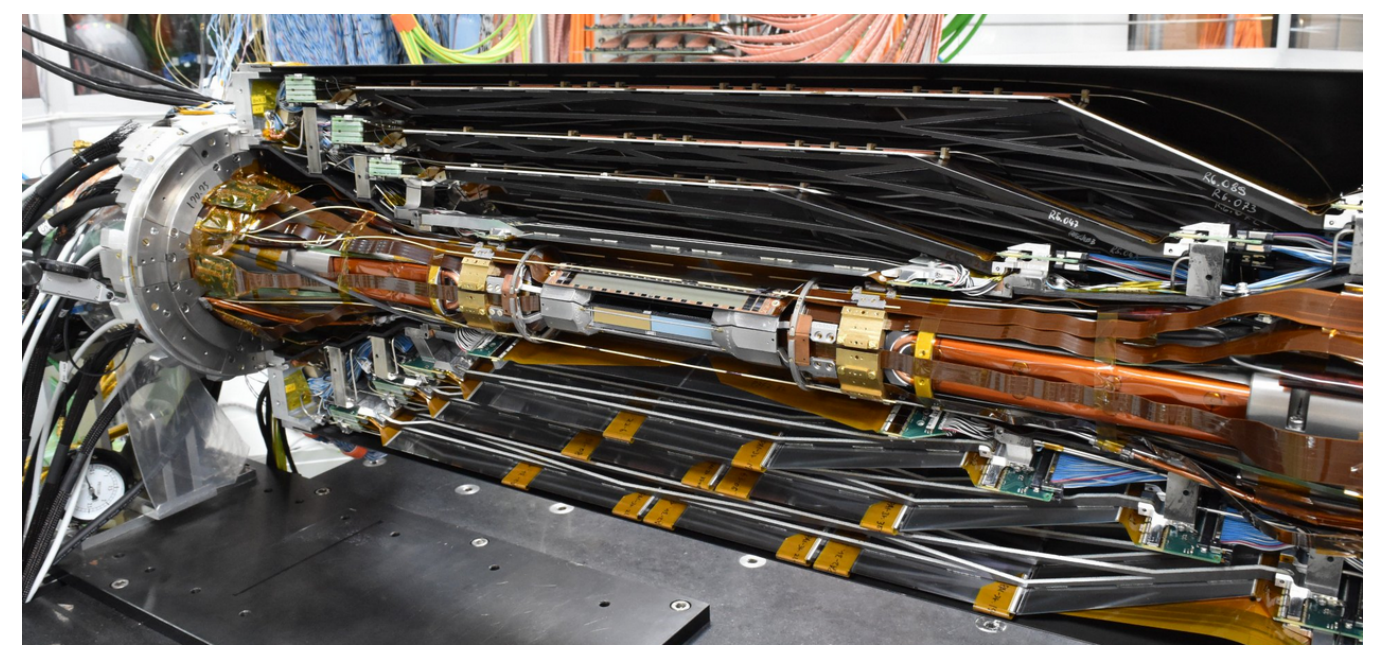

Figure 1: Half of the VXD before completion in 2018, pixel sensors are visible in the middle. The slanted SVD sensors in forward part (right) are clearly visible.

The SVD [3] is using sensors with a thickness of $300 \mu \mathrm{m}\left(0.7 \% X_{0} /\right.$ layer $)$ in its barrel part and trapezoidal slanted sensors in forward part with a thickness of $320 \mu \mathrm{m}$. Outer sensors have pitch of $75 \mu \mathrm{m}$ in $r-\phi$ and $240 \mu \mathrm{m}$ along $z$ direction. Sensors in the first layer have pitch $160 \mu \mathrm{m}$ along $z$ and $50 \mu \mathrm{m}$ in $r-\phi$ direction. The SVD has a crucial role in the operation of the VXD at higher luminosities; tracks found in the SVD are extrapolated to the PXD to define regions of interest (ROI). Only the hits in the ROIs are read out, thus reducing substantially the amount of data to process and store. Furthermore, the SVD is used for standalone particle identification and tracking of low momentum tracks that do not reach the drift chamber. The ability of the SVD to suppress beam-related background relies on its excellent hit-time resolution of approximately $3 \mathrm{~ns}$.

\section{Vertex Reconstruction at Belle II}

In this section we describe the various algorithms used in the reconstruction of tracks and vertices in the SVD, as well as its alignment. Sections 3.1, 3.2 and 3.3 describe the track finding, track and vertex fitting, and alignment, respectively.

\subsection{Track Finding}

A modular tracking design in Belle II software allows the finding, fitting and merging strategy to be adjusted to the background conditions, detector degradation, or to reconstruct cosmic ray tracks. A very simplified workflow of the standard charged-track reconstruction is sketched in Fig. 2. The track-finding stage follows after reconstruction of individual sub-detector hits, which is the clustering of VXD hits and the reconstruction of drift circles around signal wires in the drift chamber (CDC). After hit reconstruction, the tracks are found primarily in the CDC, with combining global and local methods. The global method is based on a conformal (Legendre) transformation that maps the helical trajectories of the tracks to straight lines in the conformal space. As drift circles map to circles, the task of track finding is to search for high density regions 
in the parameter space of plausible tangents to the drift circles. In comparison, the local approach looks for compatible neighbouring hits forming a track using a cellular automaton approach [4].

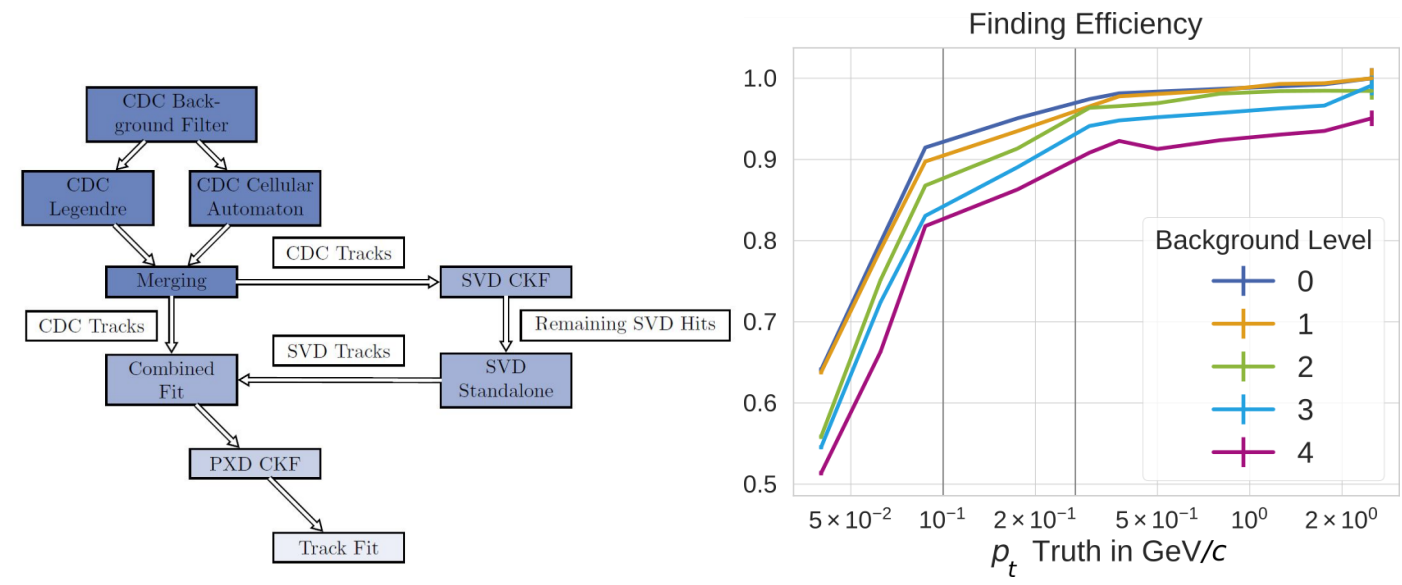

Figure 2: Left: A schematic of the tracking workflow at Belle II, starting with track-finding in the drift chamber (CDC). Right: track-finding efficiency for primary (going from interaction point) particles (factoring-out geometrical acceptance), estimated from simulated events, as a function of $p_{t}$ and background level $(0=$ no background, 1 = nominal background).

Track candidates found by global and local approach are merged and extrapolated using a Combinatorial Kalman Filter (CKF) [4]. The CKF algorithm extrapolates the tracks inwards and looks for hits to attach. The track parameter predictions are updated with each hit added. A boosted decision tree technique is used for hit filtering and duplicate removal. The CKF is also used to attach the final pixel hits to tracks extended from drift chamber to SVD, or to SVD-only tracks, found by the standalone algorithm.

The SVD-only track finding aims to reduce the combinatorics by combining space points from compatible sectors and applying filters to reject any background hits. Training of compatibility relations and filters is done with simulations, then stored in look-up tables. From the look-up tables and space points a segment network is formed for each event and a cellular automaton is utilized to find tracks as the longest paths in the network. Fake and clone tracks are removed based on multiple quality indicators.

Figure 2 shows the overall track-finding performance on simulated $\Upsilon(4 S)$ events, as a function of transverse momentum $p_{t}$ of the particles and the beam background level. The track finding efficiency (factoring-out geometrical acceptance) is greater than $90 \%$ for particles with $p_{t}>$ $100 \mathrm{MeV} / c$ at nominal background, though tracks down to $50 \mathrm{MeV} / c$ can be found with an efficiency greater than $60 \%$. The overall tracking scheme proves robustness against variations of the amount of beam background and can operate reasonably with twice the nominal background levels, even without optimization for this level of background.

\subsection{Track and Vertex Fitting}

Found tracks are fit at several stages of the reconstruction chain, to yield partial track parameters for further extrapolations. At all stages, including the final track fit, the GENFIT toolkit [5] is used. GENFIT was largely rewritten based on the experience of its use at Belle and COMPASS, 
as well as simulation studies for the PANDA experiment. It allows any measurement type to be used in the fit, while accounting for the inhomogeneous magnetic field, energy loss and material effects. GENFIT has a generic interface to several different fitters: standard Kalman fit, Deterministic Annealing Filter (DAF), and General Broken Lines (GBL) [6]. At Belle II, DAF is used as the default fit method because it allows hit down-weighting. The GBL method is used for alignment as described in Sec. 3.3.

Decay vertices are found by combining individual tracks, while applying additional conditions. Resulting combinations of particles are fit employing additional constraints for the given decay, such as known interaction point position or the hypothesised invariant mass of the decaying particle. These constraints can substantially improve the fit. At Belle II, the default vertex fitter is TreeFitter, which is a Belle II implementation of a Kalman-based global-decay-chain fit [7]. TreeFitter can use various constraints, fit neutral particles or particle lifetimes.

\subsection{Track Based Alignment}

Alignment and calibration are key ingredients required to reach the target performance of the detector. At Belle II, a single fully integrated alignment method is used for PXD, SVD, CDC and muon system. The method is based on a track refit with GBL and the Millepede II solver [8].

GBL constructs the linear equation system for the track fit and stores the output, including additional global derivatives with respect to alignment parameters and their corresponding labels. The output is then used by Millepede II, a global linearized $\chi^{2}$ minimizer for a very large number of parameters. The parameters are of two types: local and global. The local parameters correspond to parameters of individual tracks or composite objects, such as a $K_{\mathrm{S}}^{0} \rightarrow \pi^{+} \pi^{-}$decay. The global parameters of interest correspond to those related to the alignment. Millepede II utilizes the special block matrix structure of the problem to solve for the alignment parameters efficiently, keeping all correlations in the solution.

For alignment of the VXD detector, the detector is represented as half-shells, ladders and individual sensors, resembling the mechanical structure. For each component of the structure, six rigid-body-alignment parameters - translations in local object system and rotations around the local axes - are used. The surface of the silicon sensors is parametrized by two-dimensional Legendre polynomials up to $4^{\text {th }}$ order. In the future, corrections related to the Lorentz shift will be incorporated.

\section{Performance Results and Experience}

In this section, some of the first results related to VXD performance are presented, from commissioning data collected in 2018 in Sec. 4.1, first cosmics in Sec. 4.2 and physics run data in 2019 in Sec. 4.3.

\subsection{Commissioning Data}

In 2018, a commissioning run, with only one section of the vertex detector, and a commissioning of the full vertex detector with cosmic ray tracks were successfully performed. Initially large displacements (hundreds of $\mu \mathrm{m}$ ) and sensor deformations (up to $\sim 0.5 \mathrm{~mm}$ for some SVD sensors) were observed in the initial alignment results. 
After implementing sensor deformations into the alignment, as well as the respective corrections at reconstruction level, the sensors appear near to flat and the residual distributions are close to optimal, as shown in Fig. 3. Hence, including sensor deformations is crucial for good alignment, especially for SVD sensors. Deformations parametrized by $3^{\text {rd }}$ order Legendre polynomials, are determined for each silicon sensor; higher orders are not statistically significant. [10]
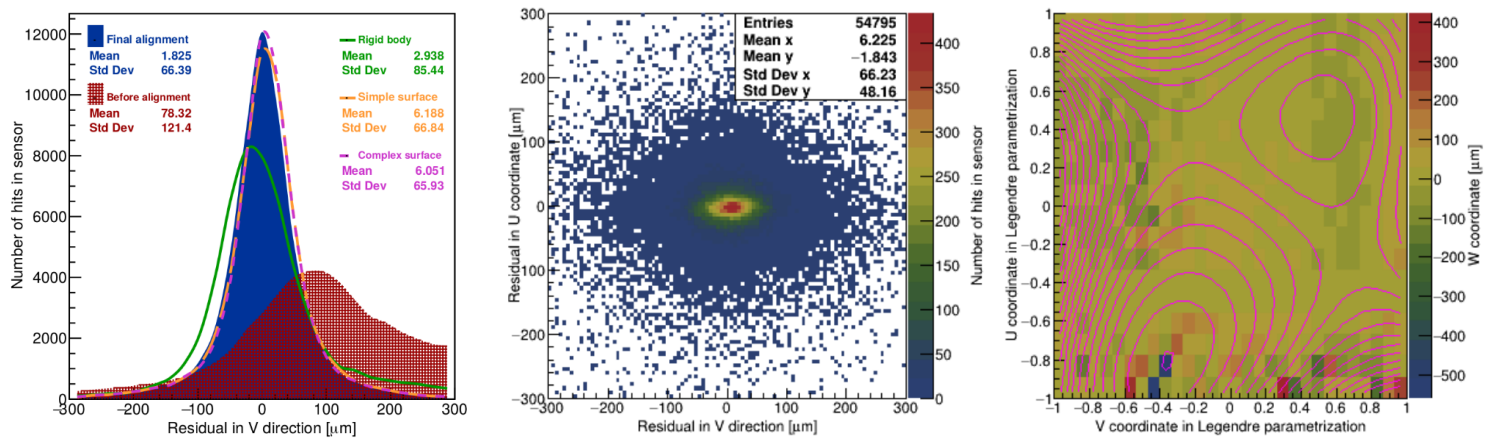

Figure 3: Data from 2018 show track-to-hit residuals in direction along the beams for one SVD sensor. One dimensional track-to-hit residuals (left) show comparison of misaligned initial geometry (red), simple rigid-body alignment (green) and alignment including sensor deformations (blue), also shown in 2D (center). Right: The deviation of the sensor from flatness (denoted as W-coordinate on color scale) after alignment can be estimated from residual dependence of non-perpendicular tracks plotted over the sensor area. The contours visualise an independent fit of the distribution after alignment with Legendre polynomial of third order (only second order Legendre polynomial is used for this particular alignment). [10]

\subsection{Cosmic Ray Data}

After VXD installation, but before the first physics run, the detector was calibrated and aligned using cosmic-ray data, initially without magnetic field. The full alignment was performed, including alignment of the CDC.

Basic standard track-to-hit residuals did not show any significant issues with alignment. However, the so called overlap residuals indicated a severe issue, see Fig. 4. These residuals are calculated using tracks crossing a silicon layer twice, hitting overlapping regions of neighbouring sensors in a layer. Overlap residuals are a powerful method to detect detector misalignment to which standard residuals and alignment are only weakly sensitive, such as radial expansion or twist deformation. It allows different systematic distortions to be qualitatively distinguished; this has been verified with simulation studies. The only disadvantage is the large statistics needed, because less than $10 \%$ of tracks have an overlap hit doublet. The 'snowman' structure in Fig. 4 (left) was reminiscent of radial expansion from MC studies on misalignment and indeed, the wrong pitch for the sensors was compensated by the alignment procedure, changing the radius of the affected layers. The issue was traced back to a wrong pitch size for SVD sensors in software. There are no indications of significant problems in overlap residuals observed after the correction.

\subsection{Physics Data}

The first collision data allowed further tests of the detector performance. Initial studies which 


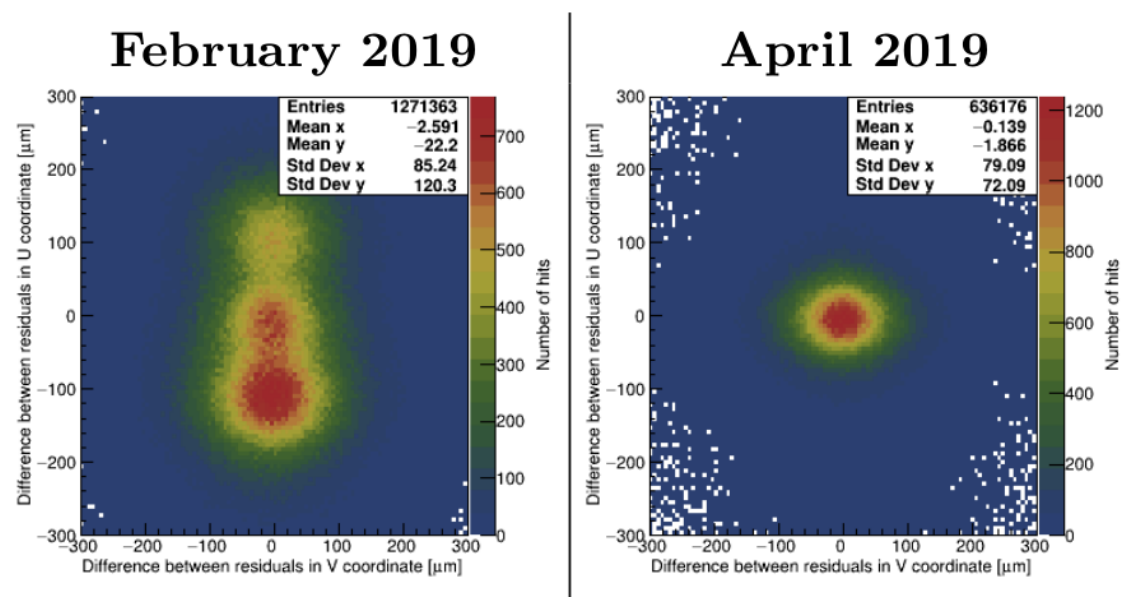

Figure 4: Overlap residual distributions from all VXD sensors in 2019 cosmic data before fixing SVD sensor pitch (left) and after (right).

targeted detector resolution, were followed by the first physics measurements, which require excellent performance of the vertex detector.

A standard method to estimate impact parameter resolution is to compare two tracks with common vertex at the point of closest approach to the origin and plot the difference of the track parameters. For example, the transverse impact parameter difference, $\Delta d_{0}$, which is shown in Fig. 5 (left).

Another method is available at Belle II, which utilizes the very small size of the luminous region in the vertical direction of $\sigma_{y} \sim 1.5 \mu \mathrm{m}$ in the initial physics runs. For nearly horizontal tracks, the variance of $d_{0}$ is an estimate of the transverse impact parameter resolution. For nearly vertical tracks, the beamspot size contribution to the resolution increases, because in the horizontal plane the size of the luminous region is about $\sigma_{x} \sim 15 \mu \mathrm{m}$. Therefore the dependence of the width of $d_{0}$ distribution on $\phi_{0}$, the angle of the track with respect to the vertical direction, is expected to follow $\sigma\left(d_{0}\right)=\sqrt{\sigma_{i}^{2}+\left(\sigma_{x} \sin \phi_{0}\right)^{2}+\left(\sigma_{y} \cos \phi_{0}\right)^{2}}$, where $\sigma_{i}$ is the actual detector resolution in the transverse impact parameter, which can be obtained from a fit.

Both methods agree within statistical errors on the transverse impact parameter resolution of $14.1 \pm 0.1 \mu \mathrm{m}$, where the uncertainty is statistical only. Also the slight discrepancy to simulation is seen consistently in both methods, which is still under investigation.

A further powerful test of the full VXD reconstruction performance, including hit reconstruction, tracking and vertexing, is measuring the $D^{0}$ lifetime using the decay chain $D^{*} \rightarrow \pi_{\text {slow }} D^{0}, D^{0} \rightarrow$ $K \pi$. The TreeFitter algorithm, discussed in Sec. 3.2, is used for a full decay chain fit, which allows the determination of the $D^{0}$ lifetime from the displacement of the $\pi_{\text {slow }}$ vertex (with the short-lived $D^{*}$ constrained to the calibrated IP position) and common $K \pi$ vertex. Figure 6 shows the topology and the fits to the $D^{0}$ invariant mass and lifetime distributions. The measured $D^{0}$ lifetime is $(370 \pm 40)$ fs, where the uncertainty is statistical, which is in agreement with the current world average $(410.1 \pm 1.5)$ fs [9]. This measurement demonstrates the ability of the VXD to reconstruct decay vertices with micron precision. 

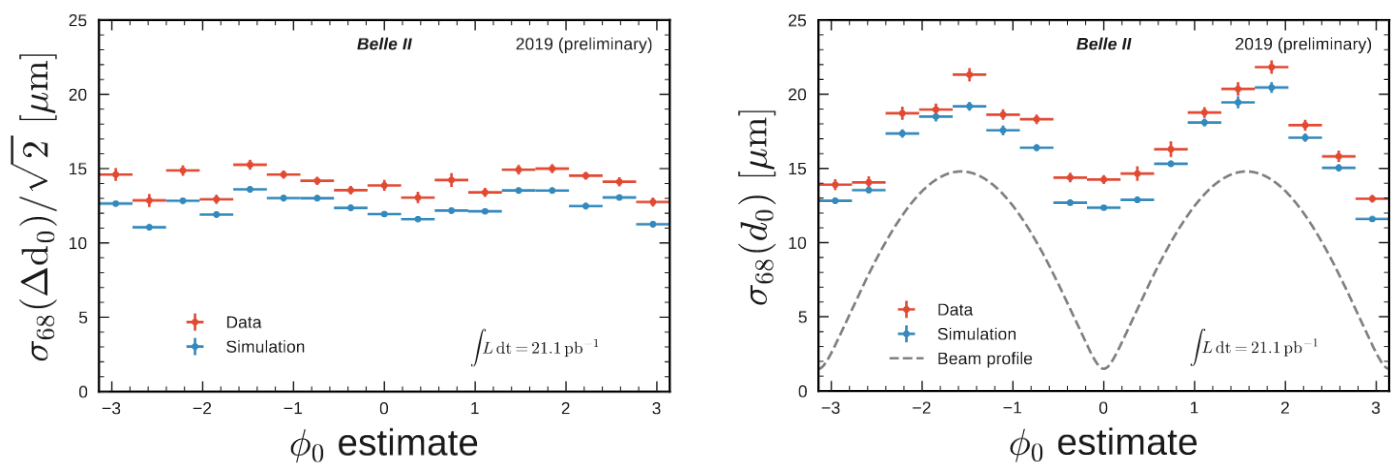

Figure 5: Transverse impact parameter resolution vs. $\phi_{0}$ of (positive) track, for dimuon and Bhabha events collected during 2019, estimated with the standard method (left) and the method utilizing the very small luminous region size in the vertical direction (right). The beam profile (dashed line, which corresponds to $\sigma_{i}=0 \mu \mathrm{m}$ ) and simulation (blue) is using horizontal and vertical size $\sigma_{x}=14.8 \mu \mathrm{m}$ and $\sigma_{y}=1.5 \mu \mathrm{m}$, respectively, calculated from machine parameters. Here $\sigma_{68}$ denotes half of the symmetric range about the median containing $68 \%$ of the distribution.
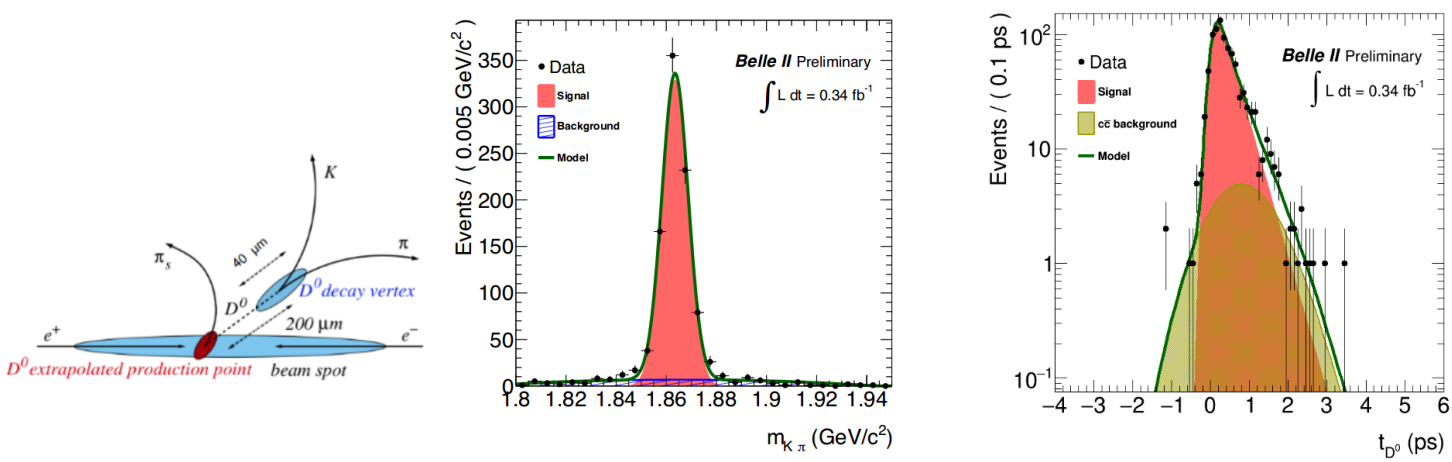

Figure 6: Left: schematics of the decay chain in which $D^{0}$ lifetime is measured. Center: invariant mass of the $K \pi$ pair. Right: distribution for the measured $D^{0}$ lifetime. The fit result is superimposed on the distribution.

\section{Conclusion}

The Belle II experiment started taking data in 2019 and aims to increase the luminosity by roughly two orders of magnitude in the coming years. The physics program requires excellent vertex reconstruction, which depends upon track finding, fitting and detector calibration and alignment. We presented a basic overview of the components and demonstrated the performance on validations and monitoring with first data, from low-level (residuals) to physics-level studies. Many more advanced analyses are still statistically limited and may eventually reveal, for example, slight alignment issues (like ignoring time-dependent movements), which will need to be addressed.

\section{References}

[1] Z. Doležal, S. Uno et al., Belle II Technical Design Report, High Energy Accelerator Research Organization (2013) arXiv:1011.0352. 
[2] C. Marinas et al. [DEPFET Collaboration], The Belle II pixel detector: High precision with low material, Nucl. Instrum. Meth. A 731 (2013) 31.

[3] K. Adamczyk et al., The silicon vertex detector of the Belle II experiment, Nucl. Instrum. Meth. A 824 (2016) 406.

[4] T. Hauth (Belle II Tracking Group), Belle II Track Reconstruction and Results from first Collisions, EPJ Web Conf. 214 (2019) 02032.

[5] J. Rauch and T. Schlüter, GENFIT - a Generic Track-Fitting Toolkit, arXiv:1410.3698.

[6] C. Kleinwort, General Broken Lines as advanced track fitting method, Nucl. Instrum. Meth. A 673 (2012) 107.

[7] W. D. Hulsbergen, Decay Chain Fitting with a Kalman Filter, Nucl. Instrum. Meth. A 552 (2005) 566.

[8] V. Blobel, C. Kleinwort, and F. Meier, Fast alignment of a complex tracking detector using advanced track models, Comp. Phys. Commun. 182 (2011) 1760.

[9] M. Tanabashi et al. (Particle Data Group), Phys. Rev. D 98 (2018) 030001

[10] J. Kandra et al. Calibration and alignment of the Belle II tracker (2019) arXiv:1910.06289v1 [physics.ins-det] 